\title{
On a Field Investigation and Open Data Analysis to Identify Diffusion Sources of Radioactive Substance
}

\author{
Kazunari Ishida
}

\begin{abstract}
After the Fukushima No. 1 nuclear power plant disaster due to the huge earthquake, spreading contamination is a serious fear for Japanese society. In this paper, a field investigation is described to understand the contamination in the west part of Japan, which is in a region approximately a thousand $\mathrm{km}$ from the east power plant. Based on the contamination origin found in the investigation, open data is analyzed to identify important factors for spreading radioactive substance in terms of relationship between air dose rate and weather conditions. Based on the analysis, a detection rule or algorithm for the contamination origin is summarized to develop a detection system for radiation warning. Air dose rate is observed on each public monitoring point. The nearest weather observation station for each public monitoring point concerning air dose rate is also identified to analyze the relationship between air dose rate and weather conditions.

According to the analysis, rainfall is an effective trigger to drop radioactive substance, such as contaminated airborne particles, to the ground. However, long-term rainfall, e.g., over half of a day, also has an effect to clean the air and ground. The most serious factor is wind direction from spreading origins of the particles in terms of air dose rate. In addition, consistent wind from a spreading origin can bring the particles without rainfall. On the other hand, wind speed seems not to be a big factor of increase of air dose rate, although the speed could multiply the effect of wind consistency on a spike of the dose rate.
\end{abstract}

Index Terms - Open data, radioactive substance, local society, weather condition, location information

\section{INTRODUCTION}

After the huge earthquake in Japan on March 11, 2011, radioactive substance derived from the Fukushima No. 1 nuclear power plant could affect the world's environment and society. Japanese people were concerned about contaminated food, wood, resources, and goods due to air and water pollution in terms of radioactive substance. According to the nature blog news on December 21 in 2012, one of the most interesting articles on social media is an academic article concerning the biological effect of the crippled Fukushima nuclear power plant [1]. Worldwide attention on this article means that the crippled nuclear power plant and diffusion of radioactive substance from the plant is a major concern and threat against our environment.

The Japanese government has a warning system, called

Manuscript received January 10, 2013; revised March 4, 2013. This research was supported in part by Grants-in-Aid for Scientific Research (C) (General) (No. 24500301, Representative: Kazunari Ishida) from Japan Society for the Promotion of Science (JSPS).

$\mathrm{K}$. Ishida is with the Faculty of Applied Information Science, Hiroshima Institute of Technology, 2-1-1 Miyake, Saeki-ku, Hiroshima, 731-5193, Japan (e-mail: k.ishida.p7@it-hiroshima.ac.jp).
SPEEDI (System for Prediction of Environmental Emergency Dose Information) [2], which is supposed to predict the radiation spread based on information concerning power plant, weather conditions, and geographic area in terms of dose rate. SPEEDI is intended to detect a serious accident at a nuclear power plant; however, it is not intended as a secondary diffusion from contaminated goods, water, and other items. In order to specify a radiation level, the Japanese government provides information collected from monitoring points in Japan [3]. This paper analyzes the relationship between radiation level and weather conditions toward development of a detection system to identify sources or origins of spreading radioactive substance.

\section{Diffusion of RADIOACTIVE SUbSTANCE}

Many researchers have been trying to clarify the environmental and social effects of radioactive substance. Yasunari et al. [4] estimated the amount of radioactive substance on and inside soil in Japan. Koyama drew a contamination map around Shizuoka prefecture due to the Fukushima No. 1 nuclear power plant [5]. Hayashi researched the contamination of wood in forests [6]. The Forestry Agency of Japan provides questions and answers concerning handling wood products because the products might be contaminated [7].

The Ministry of Agriculture of Forestry and Fisheries provides information concerning the limitation of export of Japanese products to other countries, and inspection of agricultural products and fisheries products with respect to cesium 137 contamination [8]. The Ministry of Land, Infrastructure, Transport, and Tourism of Japan provides a report on the result of inspection of drainage and sewage sludge with respect to radiation levels [9]. The Ministry of Health, Labor and Welfare provides information concerning inspection results of water supply with respect to cesium 137 contamination [10].

According to these reports, agricultural and fisheries products and water supply contain a thousand times contamination of radioactive substance compared to that before the Fukushima nuclear power plant disaster. The clean association of Tokyo 23 waste reports that ashes contain radioactive substance continuously after the disaster. In addition, burning earthquake debris derived from the northeast regions in Japan is another significant concern of spreading radioactive particles because burning earthquake debris could make secondary spreading contamination worldwide, as much as the primary spreading contamination [11], [12]. 


\section{FIELD INVESTIGATION}

To find an appropriate source of spreading a radioactive substance, a field investigation was conducted with a recordable Geiger counter and GPS in a car driven among four prefectures in the west part of Japan. The results of this investigation are summarized in Fig. 1-Fig. 6. The x-axis in each figure is the time period, where the unit is a minute. The $y$-axis in each figure is the air dose rate, where the unit is micro sievert per hour.

Between Hatsukaichi and Onomichi cities on Sanyo highway in Hiroshima prefecture, a high air dose rate area is observed around the eastern part of the prefecture (Fig. 1). Between Chiyoda region and Miyoshi city on Chugoku highway in the prefecture, a high air dose area is observed in the middle (Fig. 2). From Sanyo region in the south to Sanin region in the north, through Sanyo, Chugoku, and Hamada highways, three radiation peaks were found. South Mountain tends to get high contamination due to the direct fallout from Fukushima No.1 nuclear power plant [4].

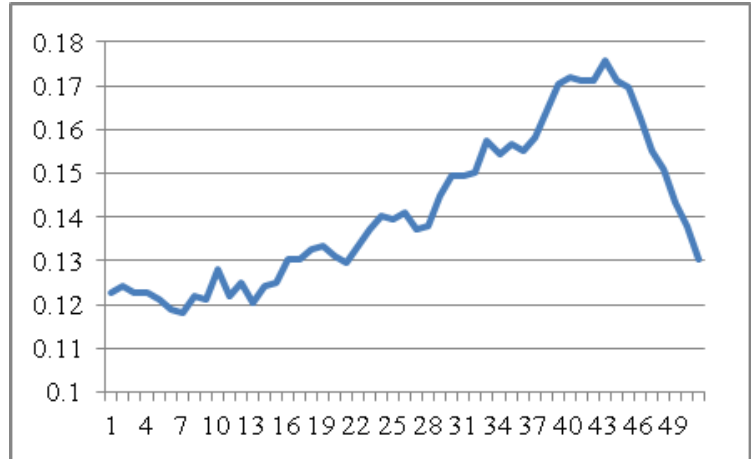

Fig. 1. Sanyo highway from west to east.

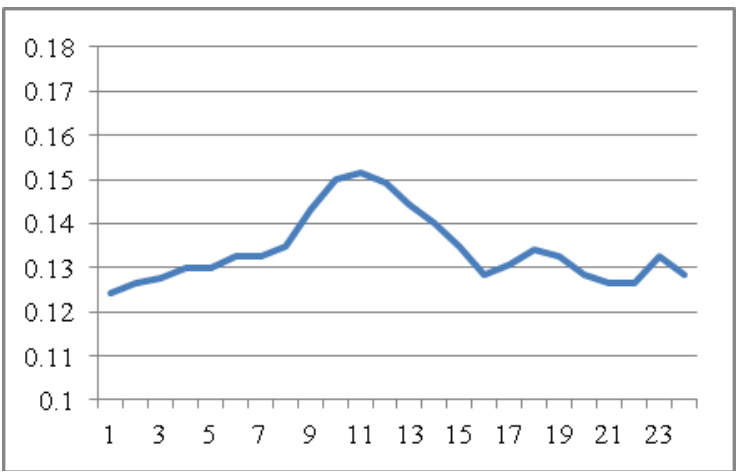

Fig. 2. Chugoku Highway from west to east.

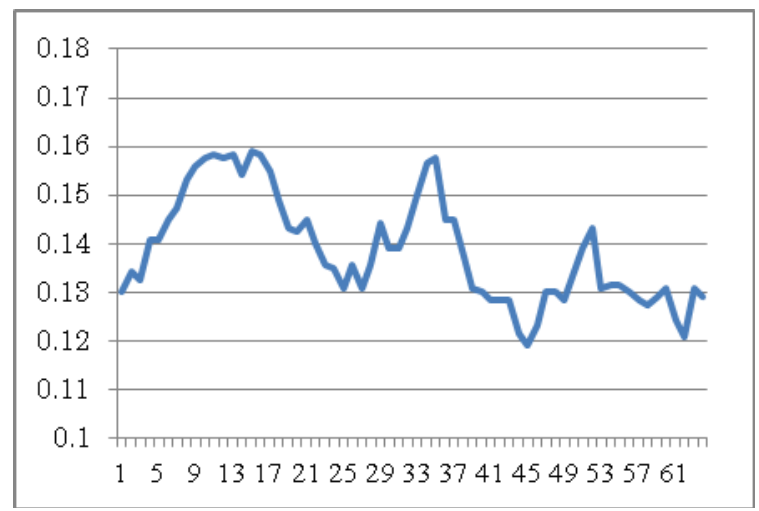

Fig. 3. Sanyo, Chugoku and Hamada Highways from south to north.

From the north part of Yamaguchi prefecture to the west part of Shimane prefecture (Fig. 4), and the west and east portions of Shimane prefecture (Fig. 5), a higher dose rate of radiation in the western part of each figure was observed compared to the eastern section. However, from the east part of Shimane prefecture to Tottori prefecture, a higher dose area appeared in the eastern section, as shown in Fig. 6.

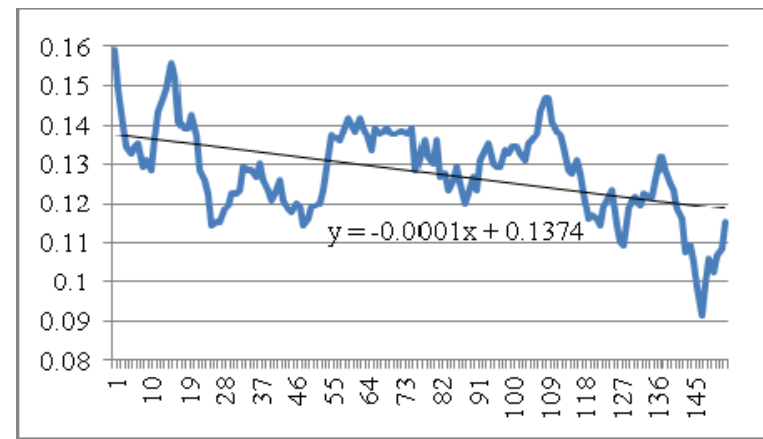

Fig. 4. Route 9 between Hagi and Hamada Cities from west to east

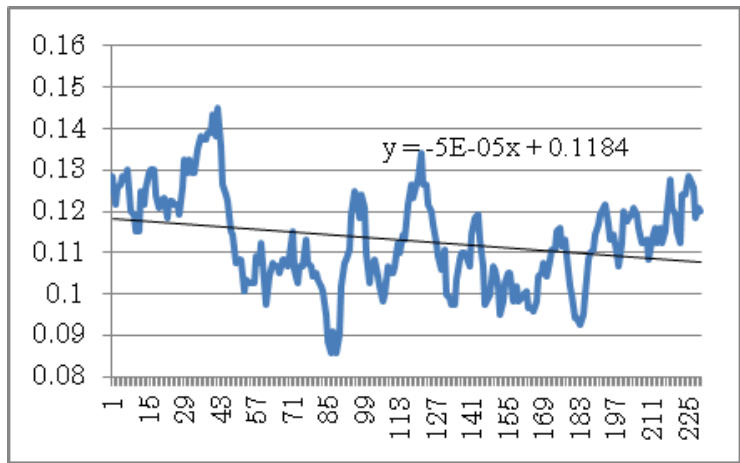

Fig. 5. Route 9 between Hamada and Matsue Cities from west to east

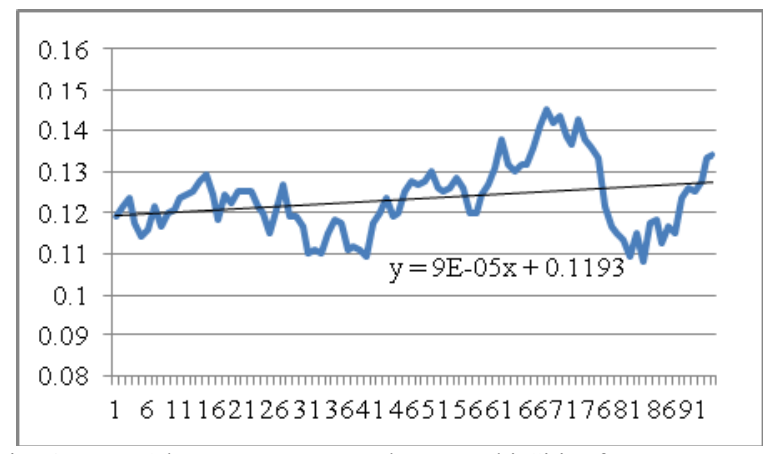

Fig. 6. Route 9 between Matsue and Kurayoshi Cities from west to east

West and east parts of Sanin region might face sources of spreading radioactive substance because this region did not get high contamination from the Fukushima fallout. Kaneyasu discussed various types of radioactive cesium, which travelled a long distance with wind blow [13]. The article means that radioactive substance can easily diffuse from a source of contamination.

One of the possible contamination sources is an incineration plant. In the west part of Japan, Kitakyushu city tried to burn earthquake debris from Ishinomaki city in Miyagi prefecture to confirm safety burning on May 23, 2011. After the confirmation, the city has started a two-year burning. In November 2012, Osaka city also tried to burn the same kind of debris from Iwate prefecture.

The Japanese government and the Ministry of Environment allow that the debris can contain radioactive substance under 100 becquerel per kiro gram. According to the FAQ of the Ministry of Environment, filtering an 
incineration plant can remove $99.9 \%$ of the substance, which means that a little substance can diffuse in the air. In this paper, Kitakyushu city was selected as an analysis area because the plants can produce radioactive airborne particles continuously for two years.

\section{OPEN DATA ANALYSIS}

Diffusion factors of radioactive airborne particles are analyzed with open data concerning air dose rate, rainfall, wind speed, and wind direction. A set of three incineration plants in Kitakyushu city were employed as safe spreading origins of radioactive airborne particles because the Japanese Ministry of Environment approved the burning earthquake debris contaminated by radioactive substance derived from the Fukushima No. 1 power plant accident.

Two monitoring posts (MPs), a Yahata common building for government offices in Kitakyushu city and a vocational training school in the western part of Yamaguchi, were also employed for analysis because they are two of the closest monitoring posts and they are located on the opposite sides from the incineration plants. The distance between the set of plants and the former MP is approximately $14 \mathrm{~km}$. The distance for the latter MP is approximately $20 \mathrm{~km}$. The former is near and the latter is far from the plants in this case. Employing an original geo-coding database, which has the same function of Google geo-coding API, the nearest weather observation station for each MP is automatically identified in terms of the name of the stations, which contains prefecture and region names. The weather station information is publicly provided by Japan's meteorological agency. In subsections A, B, and C, three cases of the former MP are explained. In subsections D and E, two cases of the latter MP are described.

\section{A. Near Consistent Wind with Rainfall}

In case 1 , consistent wind with rainfall flows from the plants to the near MP. When wind flows from an exact direction in terms of the set of incineration plants, many radioactive airborne particles are transferred from the diffusion origin. At the same time, rainfall starts, and the particles are starting to drop around MP.

Fig. 7 illustrates trajectories of air dose rate, rainfall, wind speed and consistency. The former three values are rescaled between 0 and 1 , based on the minimum and maximum values. The maximum and minimum air dose rates are 0.082 and 0.054 micro sievert per hour. The max and min amount of rainfall are 18 and $0 \mathrm{~mm}$ per hour. The max and min wind speeds are 5.7 and 1.7 meter per second. Wind consistency is defined by $(\cos (d)+1) / 2$, where $d$ is the difference between the origin's correct direction and wind direction. The range is between 0 and 1 . The unit of $\mathrm{x}$-axis is the time period where the unit is an hour. Table I summarizes the maximum and minimum values of dose rate and the three factors. The correlation coefficients between dose rate and the other factors, which are rainfall, wind speed and consistency, are $-0.09178,-0.58939$, and 0.865703 , respectively.

According to correlation coefficients, wind consistency is the most important factor on increasing the dose rate because the coefficient is 0.986 and is close to 1.0. Start of rainfall is another factor for increasing dose rate; however, continuous rainfall leads to decrease of the rate because rainfall cleans all particles in the air. This is the reason for the low or correlation coefficient between dose rate and rainfall, which is -0.09178 . Wind speed multiplies the effect of wind consistency on dose rate.

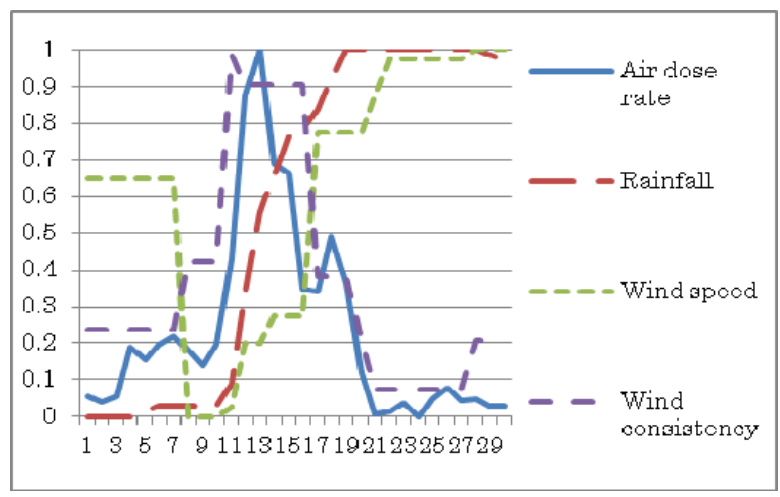

Fig. 7. Near consistent wind with rainfall.

TABLE I: MiN AND MAX IN CASE 1

\begin{tabular}{|l|r|r|}
\hline & \multicolumn{1}{|l|}{ Min } & Max \\
\hline Air dose rate & 0.058167 & 0.082 \\
\hline Rainfall & 0 & 18 \\
\hline Wind speed & 1.7 & 5.7 \\
\hline Wind consistency & 0.07368 & 0.986 \\
\hline
\end{tabular}

\section{B. Near Inconsistent Wind with Rainfall}

In case $2 \mathrm{~A}$, inconsistent wind with rainfall flows from the plants to the near MP. When wind flows different angles from the diffusion origin, the maximum air dose rate, which is 0.065 , is not very high compared to the minimum rate 0.0585 (Fig. 8). In this case, the wind consistency is between 0.095492 and 0.383 , which is quite low compared to case 1 . Table II summarizes the maximum and minimum values of dose rate and the three factors. The correlation coefficients between dose rate and the other factors, which are rainfall, wind speed and consistency, are $-0.42046,-0.2712$, and 0.13966 , respectively.

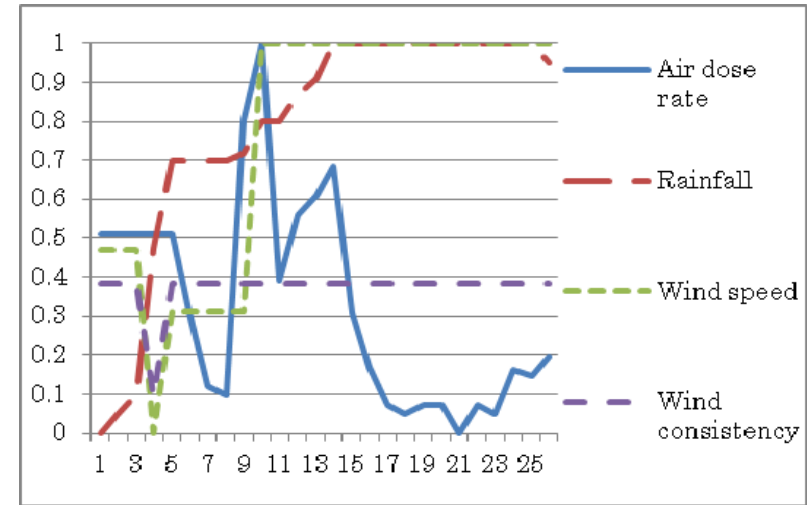

Fig. 8. Near inconsistent wind with rainfall.

TABLE II: MiN AND MAX IN CASE 2A
\begin{tabular}{|l|r|r|}
\hline & \multicolumn{1}{c|}{ Min } & \multicolumn{1}{c|}{ Max } \\
\hline Air dose rate & 0.0585 & 0.065 \\
\hline Rainfall & 0 & 10 \\
\hline Wind speed & 2.4 & 5.6 \\
\hline Wind consistency & 0.095492 & 0.383 \\
\hline
\end{tabular}




\section{Near Long-Term Consistent Wind before Rainfall}

In case $2 \mathrm{~B}$, long-term consistent wind before rainfall flows from the plants to the near MP. When consistent wind blows long term before rainfall, air dose rate reaches high value, which is 0.091333 in this case. A big spike on dose rate is observed at time period 9, when small rainfall starts (Fig. 9). The maximum dose rate is achieved when rainfall reaches the maximum level. Table III summarizes the maximum and minimum values of dose rate and the three factors. The correlation coefficients between dose rate and the other factors, which are rainfall, wind speed and consistency, are $-0.12168,-0.17734$, and 0.58694 , respectively.

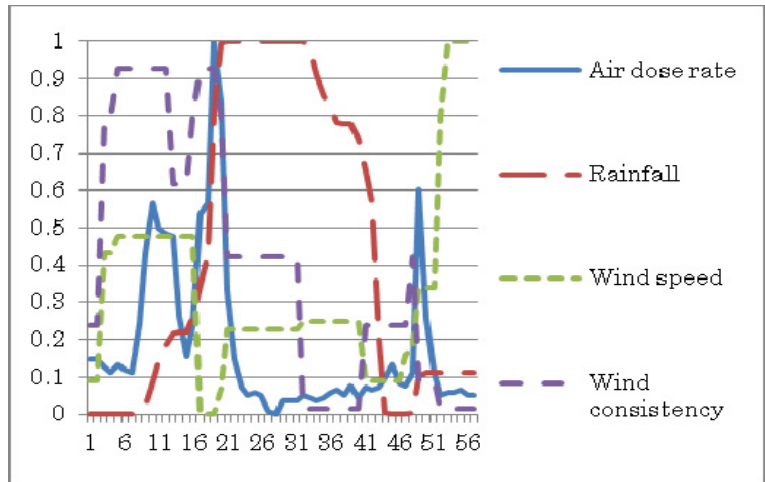

Fig. 9. Near long-term consistent wind before rainfall.

TABLE III: MIN AND MAX IN CASE 3

\begin{tabular}{|l|r|r|}
\hline & \multicolumn{1}{|c|}{ Min } & \multicolumn{1}{c|}{ Max } \\
\hline Air dose rate & 0.067833 & 0.091333 \\
\hline Rainfall & 0 & 18 \\
\hline Wind speed & 2.6 & 7 \\
\hline Wind consistency & 0.013815 & 0.92632 \\
\hline
\end{tabular}

\section{Far Inconsistent Wind with Rainfall}

In case $3 \mathrm{~A}$, inconsistent wind with rainfall flows from the plants to the far MP. When wind flows different angles from the diffusion origin, the maximum air dose rate, which is 0.075 , is not very high compared to the minimum rate 0.0552 .

In this case, the wind consistency until time period 25 is almost 0 (Fig. 10). After rainfall drops almost all particles in the air, the wind direction becomes consistent with the diffusion origin. However, the consistent wind leads to a small spike of dose rate at time period 42 , even though the correlation coefficient is quite low. The correlation coefficients between dose rate and the other factors, which are rainfall, wind speed and consistency, are 0.1557, -0.009, and 0.113 , respectively. Table IV summarizes the maximum and minimum values of dose rate and the three factors.

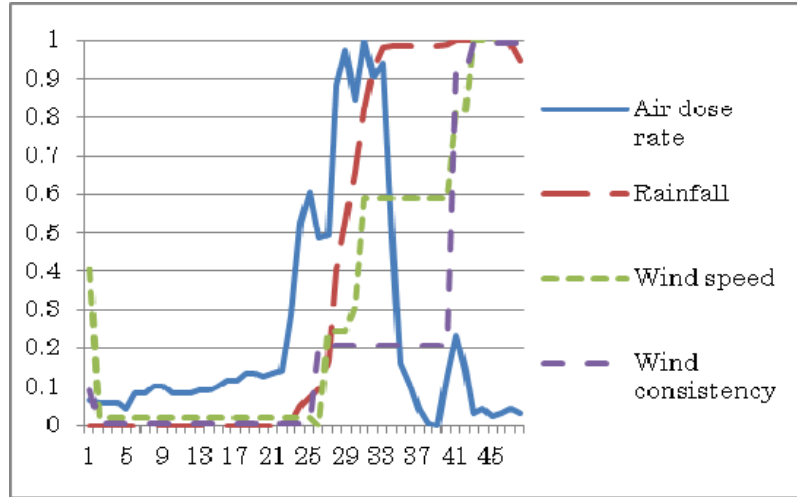

Fig. 10. Far inconsistent wind direction with rainfall.
TABLE IV: MIN AND MAX IN CASE 3A

\begin{tabular}{|l|r|r|}
\hline & \multicolumn{1}{|c|}{ Min } & \multicolumn{1}{c|}{ Max } \\
\hline Air dose rate & 0.0552 & 0.075 \\
\hline Rainfall & 0 & 38 \\
\hline Wind speed & 5.5 & 10.4 \\
\hline Wind consistency & 0.0069 & 0.9931 \\
\hline
\end{tabular}

\section{E. Far Long-Term Consistent Wind before Rainfall}

In case $3 \mathrm{~B}$, long-term consistent wind before rainfall flows from the plants to the near MP. When consistent wind blows long term before rainfall, the air dose rate is slightly high, which is 0.0792 , compared to case $3 \mathrm{~A}$. A small spike appears on time period 7 before rainfall starts because of the consistent wind (Fig. 11). Table V summarizes the maximum and minimum values of dose rate and the three factors. The correlation coefficients between dose rate and the other factors, which are rainfall, wind speed and consistency, are $-0.176,0.567$, and -0.444 , respectively.

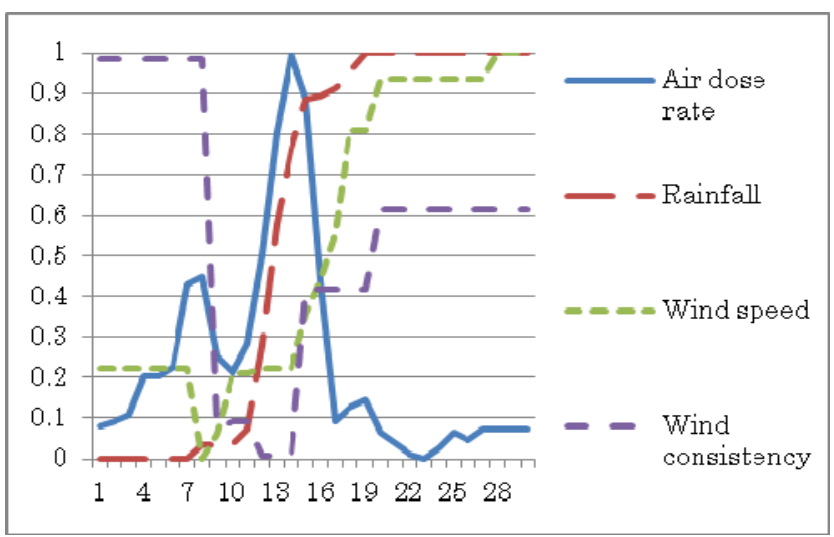

Fig. 11. Far long-term consistent wind before rainfall

TABLE V: MiN AND MAX IN CASE 3B

\begin{tabular}{|l|r|r|}
\hline & \multicolumn{1}{l|}{ Min } & \multicolumn{1}{l|}{ Max } \\
\hline Air dose rate & 0.054 & 0.0792 \\
\hline Rainfall & 0 & 14 \\
\hline Wind speed & 1.5 & 11 \\
\hline Wind consistency & 0.0069 & 0.98719 \\
\hline
\end{tabular}

\section{DISCUSSION}

In this section, several results are summarized to develop a detection system to find spreading sources of radioactive particles.

In all five cases, rainfall is an important trigger to drop the particles to the ground to contaminate. However, long-term rainfall, e.g., over half of a day, also has an effect to clean the air and ground. This means that sunny regions with short rainfall might suffer from an accumulation of radioactive particles.

The most serious factor is wind direction from spreading origins, e.g., case 1, case $2 \mathrm{~B}$ and case $3 \mathrm{~B}$, in terms of air dose rate. Consistent wind from a spreading origin can bring radioactive particles without rainfall, as illustrated around time period 5 in Fig. 11. On the other hand, wind speed seems not to be a big factor of increase of air dose rate, although the speed could multiply the effect of wind consistency on spike 
of the dose rate.

Unfortunately, the two MPs always detect some amount of radioactive particles when rainfall starts with any wind direction because they are very close from the set of inclination plants. Hence the data from other MPs might have to be analyzed to understand the diffusion process in detail. However, the next step of this research is to develop a prototype system to detect diffusion origins, based on the findings, because of the significant concern on radiation sickness in Japan.

The system should detect spikes or a huge increase of air dose rate in the first step. And then it identifies wind directions on the MPs, which observe high dose rate in the second step. After collecting a sufficient amount of data of the wind direction with location information of MPs, it searches all possible spreading origins in the third step.

\section{CONCLUSION}

This research discussed basic ideas how to develop a detecting system to find spreading radioactive substance. Spreading radioactive substance could be a major concern worldwide. In section II, this paper summarized the contamination derived from the Fukushima No. 1 nuclear power plant in Japan. To find an appropriate analysis area of spreading radioactive substance, a field investigation was conducted with a survey meter on an automobile. Around the found area, data concerning air dose rate and weather conditions are analyzed on two MPs near the spreading area. Based on the findings from the analysis, this research will provide a prototype system to detect spreading origins of radioactive substance.

\section{REFERENCES}

[1] A. Hiyama, C. Nohara, S. Kinjo, W. Taira, S. Gima, A. Tanahara, and J. M. Otaki, "The biological impacts of the Fukushima nuclear accident on the pale grass blue butterfly," Nature Scientific Reports, Article number 570, August 9, 2012.

[2] H. Terada, H. Nagai, A. Furuno, T. Kakefuda, T. Harayama, and M Chino, "Development of Worldwide Version of System for Prediction of Environmental Emergency Dose Information," WSPEEDI 2nd Version, vol. 7, No. 3, pp. 257 - 267, 2008 (in Japanese).

[3] Ministry of Education, Culture, Sports, Science and Technology of Japan, "White Paper on Education, Culture, Sports, Science and
Technology, Fiscal Year 2011,” Saiki Printing Co., Ltd., Jun 29, 2012 (in Japanese).

[4] T. J. Yasunari, A. Stohl, R. S. Hayano, J. F. Burkhart, S. Eckhardt, and T. Yasunari, "Cesium-137 deposition and contamination of Japanese soils due to the Fukushima nuclear accident," PNAS, vol. 108, no. 49, pp. 19530 - 19534, December 6, 2011.

[5] M. Koyama, "Making detail map of radioactive contamination around Shizuoka prefecture due to the Fukushima nuclear power plant disaster," Japan Society for Disaster Information Studies, $14^{\text {th }}$ Domestic Conference, University of Tokyo, Oct. 27 - 28, 2012 (in Japanese)

[6] T. Hayashi, "Analyzing radioactive Cesium inside of Wood," Radioisotope Center News of the University of Tokyo, vol. 42, no. 4, 2012 (in Japanese)

[7] Forest Agency of Japan, "Annual Report on Forest and Forestry in Japan, Fiscal Year 2011,” ZENRINKYOU, April 27, 2012 (in Japanese).

[8] Ministry of Agriculture, Forestry and Fisheries of Japan, "Fiscal Year 2011 Annual Report on Food, Agriculture and Rural Areas in Japan," Saiki Printing Co., Ltd., May 21, 2012 (in Japanese).

[9] Ministry of Land, Infrastructure, Transport and Tourism of Japan, "Fiscal Year 2011 Annual Report on Land, Infrastructure, Transport and Tourisms in Japan," GYOSEI Co., July 9, 2012 (in Japanese).

[10] Ministry of Health, Labor and Welfare of Japan, "Fiscal Year 2011 Annual Report on Health, Labor and Welfare in Japan," Nikkei Printing Inc., September 2012 (in Japanese).

[11] W. A. Bird and E. Grossman, "Chemical Aftermath: Contamination and Cleanup Following the Tohoku Earthquake and Tsunami," Environ Health Perspect, vol. 119 no. 7, pp. 290 - 301 July 1, 2011.

[12] J. D. Leona, D. A. Jaffe, J. Kaspar, A. Knecht, M. L. Miller, R.G.H. Robertson, and A. G. Schubert, "Arrival time and magnitude of airborne fission products from the Fukushima, Japan, reactor incident as measured in Seattle, WA, USA," Journal of Environmental Radioactivity, vol. 102, no. 11, pp. 1032-1038, November 2011.

[13] N. Kaneyasu, H. Ohashi, F. Suzuki, T. Okuda, and F. Ikemori, "Sulfate Aerosol as a Potential Transport Medium of Radiocesium from the Fukushima Nuclear Accident," Environmental Science \& Technology, vol. 46, no. 11, pp. 5720-5726, June 5, 2012.

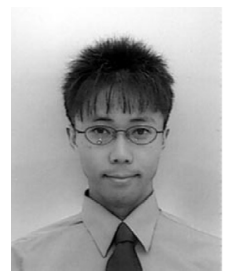

Kazunari Ishida received the B.Eng. degree from Toyohashi University of Technology Toyohashi, Japan, in 1993 and the M.Eng. and D.Eng. degrees from the University of Electro-Communications Tokyo, Japan, in 1995 and 1998, respectively.

$\mathrm{He}$ is currently an associate professor with the Faculty of Applied Information Science, Hiroshima Institute of Technology. His publications have appeared in IEEE Transactions on System, Man, and Cybernetics, proceedings of the WWW conference and in other English and Japanese academic journals.

He has been working on developing web and mobile applications and analyzing social media. He is also working on analyzing social phenomena with mathematical and computer simulation models. 